\title{
RESIDENTIAL ELECTRICITY RATES FOR THE UNITED STATES FOR SOLCOST DATA BANK CITIES
}

Prepared by

Laurie E. Smith

May 1981

Prepared for
U.S. DEPARTMENT OF ENERGY

under contract DE-AC02-79CS30166 


\section{DISCLAIMER}

This report was prepared as an account of work sponsored by an agency of the United States Government. Neither the United States Government nor any agency Thereof, nor any of their employees, makes any warranty, express or implied, or assumes any legal liability or responsibility for the accuracy, completeness, or usefulness of any information, apparatus, product, or process disclosed, or represents that its use would not infringe privately owned rights. Reference herein to any specific commercial product, process, or service by trade name, trademark, manufacturer, or otherwise does not necessarily constitute or imply its endorsement, recommendation, or favoring by the United States Government or any agency thereof. The views and opinions of authors expressed herein do not necessarily state or reflect those of the United States Government or any agency thereof. 


\section{DISCLAIMER}

Portions of this document may be illegible in electronic image products. Images are produced from the best available original document. 


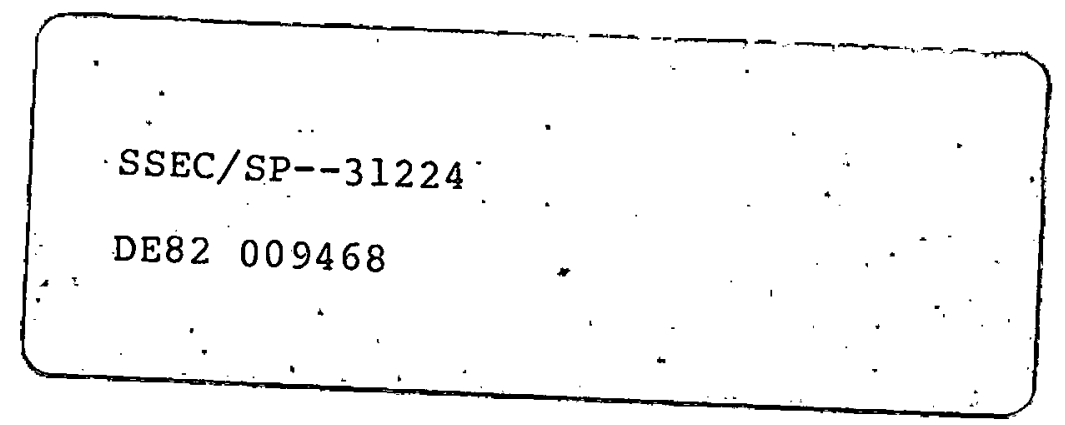

\section{RESIDENTIAL ELECTRICITY RATES FOR THE UNITED STATES FOR SOLCOST DATA BANK CITIES}

(Source: University of Alabama in Huntsville)

by

Laurie E. Smith

Planning and Evaluation

May, 1981

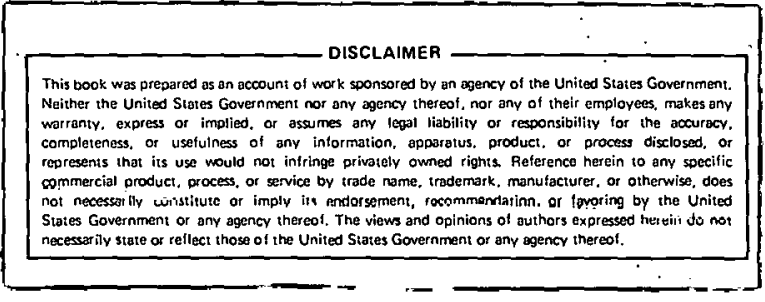


This report presents the most currently available residential electricity price information provided in the Residential Utility Rate Guide for Solcost Data Bank Cities, by Sue B. Weir, Principal Investigator; prepared for the U.S. Department of Energy Grant Number DE-FG01-79CS30013.

The format of the report is organized with the SSEC region first, followed by the rest of the nation. Amounts are given in $\$ / \mathrm{kWh}$ and $\$ / M M$ Btu for an average residence that uses 1000 Kilowatt hours a month. 
Electricity Rates For Solcost Data Cities

(Based on Monthiy Use of $1000 \mathrm{kwh}$ )

State/City

SSEC Region

Al abama

Birmingham

Huntsville

Mobile

Montgomery

Arkansas

Ft. Smith

Little Rock

Delaware

WiTmington

Florida

Apalachicola

Daytona Beach

Gainesville

Jacksonville

Key West

Miami

Orlando

Pensacola

Tallahassee

Tampa

West Palm Beach

Georgia

Atlanta

Augusta

Griffin

Macon

Savannah

Kentucky

AshTand

Covington

Lexington

Louisville

Louisiana

Baton Rouge

Lake Charles

New Orleans

Shreveport
$\$ /$ kWh

.0605

.0362

.0605

.0605

.0312

.0610

.0796

.0641

.0520

.0492

.0908

.0521

.0533

.0595

.0673

.0619

.0605

.0524

.0439

.0435

.0487

.0457

.0601

.0478

.0501

.0480

.0428

.0492

.0492

.0598

.0442
$\$ / M M B t u$

17.73

10.59

17.73

17.73

9.14

17.87

23.32

$11 / 80$

18.77

15.24

14.42

26.60

15.27

15.62

17.45

19.72

18.14

17.73

15.36

12.86

12.76

14.28

13.40

17.61

$11 / 81$

$12 / 80$

$11 / 80$
$11 / 80$
$11 / 80$
$11 / 80$

$11 / 80$
$11 / 80$
$11 / 80$
$11 / 80$

$11 / 80$
$11 / 80$
$11 / 80$
$11 / 80$

Date

$10 / 80$

$10 / 80$

$10 / 80$

$12 / 80$

$10 / 80$

$12 / 80$

$11 / 80$

$12 / 80$

$11 / 80$

$12 / 80$

$10 / 80$

$11 / 80$

$12 / 80$

$10 / 80$

$11 / 80$

$12 / 80$

14.03

$12 / 80$

$10 / 80$

$11 / 80$

$12 / 80$

14.07

12.56

14.42

14.42

17.54

12.96
$11 / 80$

$11 / 80$

$11 / 80$

$11 / 80$ 
State/City

SSEC Region

Maryland

Annapolis

Baltimore

Cumberland

Patuxent River

Sali sbury

Silver Hill

Mississippi

Jackson

Meridian

Vicksburg

North Carolina

Asheville

Cape Hatteras

Charlotte

Greensboro

Raleigh

Ok 7 ahoma

OkTahoma city

Stillwater

Tulsa

South Carolina

Charleston

Columbia

Greenville

Tennessee

Chattanooga

Knoxville

Memphis

Nashville

Oak Ridge

Texas

Abitene

Amarillo

Austin

Big Springs

Brownsville

Corpus Christi

Dallas

De] Rio

El Paso

Fort Worth
$\$ /$ kWh

.0588

.0588

.0521

.0647

.0730

.0597

.0650

.0532

.0650

.0474

.0761

.0451

.0451

.0474

.0578

.0726

.0497

.0520

.0520

.0453

.0408

.0381

.0392

.0366

.0353

.0534

.0653

.0340

.0554

.0753

.0753

.0585

.0753

.0676

.0554
$\$ / M M B t u$

Effective

Date

17.24

17.24

15.26

18.96

21.39

16.96

$11 / 80$

$11 / 80$

$11 / 80$

$11 / 80$

$11 / 80$

$11 / 80$

19.04

15.58

19.94

$11 / 80$

$11 / 80$

$10 / 80$

13.88

22.29

13.20

13.20

13.89

$10 / 80$

$10 / 80$

$11 / 80$

$11 / 80$

$10 / 80$

16.93

$10 / 80$

21.28

14.58

$10 / 80$

$10 / 80$

15.24

15.24

13.27

$11 / 80$

$11 / 80$

$11 / 80$

11.95

$12 / 80$

$11 / 80$

$11 / 80$

$11 / 80$

$11 / 80$

10.74

10.36

15.65

19.14

9.96

16.23

22.08

22.08

17.13

22.08

19.82

16.23
$11 / 80$

$11 / 80$

$12 / 80$

$11 / 80$

$11 / 80$

$11 / 80$

$11 / 80$

$11 / 80$

$11 / 80$

$11 / 80$ 
State/City

SSEC Region

Galveston

Houston

Kingsville

Laredo

Lubbock

Lufkin

Midland

Port Arthur

San Angelo

San Antonio

Sherman

Waco

Wichita Falls

Virginia

Norfolk

Richmond

Roanoke

West Virginia

Charleston

Elkins

Hinton

Huntington

Parkesburg
$\$ / \mathrm{kWh}$

.0464

.0464

.0688

.0688

.0661

.0582

.0554

.0516

.0361

.0487

.0582

.0582

.0554

.0677

.0655

.0566

.0449

.0513

.0501

.0458

.0504
$\$ /$ MMBtu

13.59

13.59

20.16

20.16

19.37

17.04

16.23

15.13

10.58

14.27

17.04

17.04

16.23

19.85

19.20

16.59

13.18

15.03

14.68

13.43

14.77
Effective

Date

$11 / 80$

$11 / 80$

$11 / 80$

$11 / 80$

$11 / 80$

$11 / 80$

$11 / 80$

$12 / 80$

$11 / 80$

$11 / 80$

$11 / 80$

$11 / 80$

$11 / 80$

$11 / 80$

$11 / 80$

$11 / 80$

$11 / 80$

$11 / 80$

$11 / 80$

$11 / 80$

$11 / 80$ 
State/City

Outside SSEC Region $\$ / \mathrm{kWh}$

$\$ /$ MMBtu

Effective

Alaska

Annette

.0471

13.80

38.21

.1304

110.46

.1039

.0826

.0708

.0465

.1583

.1979

.0693

Mantanusku

.2534

Nome

.2157

30.44

24.20

20.74

13.62

46.38

57.98

20.30

74.25

63.20

Date

Yakutat

.0659

.0626

.0659

.0683

.0654

Arizona

Phoenix

Prescott

Tucson

Winstow

.0659

19.31

18.34

19.31

20.01

19.16

19.31

$12 / 80$

$12 / 80$

$12 / 80$

$12 / 80$

$12 / 80$

$12 / 80$

$12 / 80$

$12 / 80$

$12 / 80$

$12 / 80$

$12 / 80$

$12 / 80$

Yuma

.0423

12.39

12.39

20.92

.0714

.0714

20.92

.0423

12.39

.0714

.0423

20.92

12.39

.0423

12.39

$.0714 \quad 20.92$

.0714

.0729

.0253

20.92

21.36

7.41

Mt. Shasta

.0384

.0423

Oakland

11.25

12.39

.0693

20.30

.0714

Point Mugu

20.92

.0423

.0678

Riverside

Sacramento

.0216

12.39

19.86

6.33

.1010

29.59

.0423

San Franciso

12.39

.0423

12.39

.0423

Santa Maria

12.39

.0433

$10 / 80$

$10 / 80$

$10 / 80$

$10 / 80$

$10 / 80$

$10 / 80$

Sunnyvale

12.39

$11 / 80$

$11 / 80$

$11 / 80$

$11 / 80$

$11 / 80$

$11 / 80$

$11 / 80$

$11 / 80$

$11 / 80$

$11 / 80$

$11 / 80$

$11 / 80$

$11 / 80$

$11 / 80$

$11 / 80$

$11 / 80$

$11 / 80$

$11 / 80$

$11 / 80$

$11 / 80$

$11 / 80$

$11 / 80$

$11 / 80$

$11 / 80$ 
State/City

Outside SSEC Region $\$ / \mathrm{kWh}$

Colorado

Boulder

Colorado Springs

Denver

Eagle

Grand Junction

Grand Lake

Pueblo

Connecticut

Hartford

Hawa i

Honolulu

Idaho

Boise

Lewiston

Pocatello

Twin Falls

Illinois

Cairo

Chicago

Lemont

Mol ine

Peoria

Springfield

Indiana

Evansville

$\mathrm{Ft}$. Wayne

Indianapol is

South Bend

Iowa

Ames

Burlington

Des Moines

Dubuque

Mason City

Sioux City

Kansas

Concordia

Dodge City

Goodl and

Manhattan

Topeka

Wichita
.0504

.0520

.0600

.0475

.0564

.0426

.0523

.0629

.0792

.0315

.0194

.0315

.0315

.0468

.0720

.0624

.0542

.0587

.0419

.0519

.0492

.0473

.0492

.0705

.0492

.0582

.0588

.0589

.0708

.0405

.0409

.0550

.0420

.0451

.0443
$\$ / M M B t u$

Effective

Date

14.77

$10 / 80$

15.25

$10 / 80$

17.58

$10 / 80$

13.92

$10 / 80$

$10 / 80$

12.48

$10 / 80$

15.38

$12 / 80$

18.40

$11 / 80$

23.21

$10 / 80$

9.23

$10 / 80$

5.68

9.23

9.23

$10 / 80$

$10 / 80$

$10 / 80$

13.71

$10 / 80$

21.09

18.28

$11 / 80$

$10 / 80$

15.88

$10 / 80$

$10 / 80$

17.20

12.27

$10 / 80$

15.21

14.42

13.86

14.42

$10 / 80$

$10 / 80$

$10 / 80$

$10 / 80$

20.66

14.42

17.05

17.23

17.26

20.74

$10 / 80$

$10 / 80$

$10 / 80$

$10 / 80$

$10 / 80$

$10 / 80$

11.86

$10 / 80$

$10 / 80$

$10 / 80$

$10 / 80$

$10 / 80$

$10 / 80$ 
Maine

Bangor

Caribou

Eastport

Portland

Massachusetts

Amherst $\quad 0980$

Blue Hill (Milton) $\quad .0986$

Boston

Lynn

Natick

.0713

.0620

.0713

.0582

.0986

.0819

.0986

Michigan

Alpena

Detroit

East Lansing

Flint

Grand Rapids

Houghton

Lansing

Marquette

Sault Ste. Marie

Traverse City

Minnesota

Duluth

International Falls

Minneapolis

Rochester

St. Cloud

Missouri

Columbia

Kansas City

Springfield

St. Louis

Montana

Billings

Cut Bank

Dillon

Gi asgow

Great Falls

Havre City

Helena

Kal i spell

Lewiston

Miles City

Missoula
.0456

.0599

.0535

.0614

.0614

.0649

.0535

.0555

.0342

.0526

.0547

.0359

.0518

.0458

.0533

.0525

.0726

.0554

.0454

.0271

.0302

.0271

.0271

.0271

.0271

.0271

.0242

.0271

.0439

.0271
20.89

18.16

20.89

17.05

28.71

28.88

28.88

23.99

28.88

13.36

17.55

15.67

17.99

17.99

19.02

15.67

16.26

10.02

15.41

16.03

10.52

15.17

13.42

15.62

15.38

21.27

16.23

13.30

7.94

8.85

7.94

7.94

7.94

7.94

7.94

7.09

7.94

12.86

7.94
Date

$10 / 80$

$10 / 80$

$10 / 80$

$11 / 80$

$01 / 81$

$12 / 80$

$12 / 80$

$11 / 80$

$12 / 80$

$11 / 80$

$11 / 80$

$11 / 80$

$11 / 80$

$11 / 80$

$10 / 80$

$10 / 80$

$11 / 80$

$10 / 80$

$12 / 80$

$11 / 80$

$11 / 80$

$11 / 80$

$11 / 80$

$11 / 80$

$11 / 80$

$11 / 80$

$11 / 80$

$11 / 80$

$11 / 80$

$11 / 80$

$11 / 80$

$11 / 80$

$11 / 80$

$11 / 80$

$11 / 80$

$11 / 80$

$11 / 80$

$11 / 80$

$11 / 80$ 
State/City

Outside SSEC Region \$/kWh

Nebraska

Grand Island

Lincoln

North Omaha

North Platte

Scotts Bluff

Nevada

ETko

Ely

Las Vegas

Lovelock

Reno

Tonopah

Wi nnemucca

New Hampshire

Concord

New Jersey

Atlantic City

Lakehurst

Newark

Trenton

New Mexico

Atbuquerque

Claxton

Farmington

Roswell

Truth or Consequences

Tucumcari

Zuni

New York

Albany

Binghampton

Buffalo

Canton

Ithaca

Massena

New York City

Rochester

Schenectady

Syracuse
.0679

.0899

.0390

.0476

.0567

.0279

.0422

.0430

.0749

.0749

.0606

.0602

.0772

.0772

.0762

.0850

.0850

.0744

.0756

.0685

.0697

.0453

.0597

.0454

.0462

.0616

.0486

.0475

.0603

.0475

.1039

.0598

.0462

.0462
$\$ / M M B t u$

Effective

Date

19.89

$11 / 80$

$11 / 80$

$11 / 80$

11.43

13.95

16.61

$11 / 80$

$11 / 80$

8.17

12.36

12.60

21.94

21.94

17.75

17.64

$11 / 80$

$11 / 80$

$11 / 80$

$11 / 80$

$11 / 80$

$11 / 80$

$11 / 80$

22.62

$10 / 80$

22.62

22.33

24.91

24.91

$10 / 80$

$10 / 80$

$11 / 80$

$11 / 80$

46.70

$11 / 80$

22.15

20.07

17.49

13.27

17.49

13.30

$11 / 80$

$11 / 80$

$11 / 80$

$11 / 80$

$11 / 80$

$11 / 80$

13.54

18.05

14.24

13.92

17.66

13.92

30.44

17.52

13.54

13.54
$11 / 80$

$11 / 80$

$11 / 80$

$11 / 80$

$11 / 80$

$11 / 80$

$11 / 80$

$10 / 80$

$11 / 80$

$11 / 80$ 
North Dakota

Bismark

.0446

.0547

.0421

Fargo

Minot

Williston

Ohio

Akron

Cincinnati

Cleveland

Columbus

Dayton

Put-in-Bay

Toledo

Youngstown

Oregon

Astoria

Baker

Burns

Corvallis

Medford

North Bend

Pendleton

Portland

Redmond

Roseburg

Salem

Pennsylvania

AlTentown

Avoca

Erte

Harrisburg

Philadelphia

Pittsburgh

Scranton

State College

Ritode Island

Block IsTand

Newport

Providence
.0604

.0414

.0446

.0440

.0625

.0653

.0673

.0604

.0734

.0604

.0341

.0252

.0252

.0341

.0341

.0341

.0341

.0361

.0341

.0341

.0361

.0503

.0503

.0523

.0503

.1061

.0679

.0534

.0467

.1924

.0803

.0864
13.07

16.03

12.34

12.13

13.07

17.70

12.89

18.31

19.13

19.78

17.70

21.51

17.70

9.99

7.38

7.38

9.99

9.99

9.99

9.99

10.58

9.99

9.99

10.58

14.74

14.74

15.32

14.74

31.08

19.89

15.65

13.68

56.37

23.59

25.32
$11 / 80$

$11 / 80$

$11 / 80$

$11 / 80$

$11 / 80$

$12 / 80$

$11 / 80$

$12 / 80$

$12 / 80$

$12 / 80$

$12 / 80$

$11 / 80$

$12 / 80$

$11 / 80$

$11 / 80$

$11 / 80$

$11 / 80$

$11 / 80$

$11 / 80$

$11 / 80$

$11 / 80$

$11 / 80$

$11 / 80$

$11 / 80$

$11 / 80$

$11 / 80$

$11 / 80$

$11 / 80$

$11 / 80$

$11 / 80$

$11 / 80$

$11 / 80$

$11 / 80$

$11 / 80$

$10 / 80$ 
State/City

Outside SSEC Region \$/kWh

$\$ /$ MMBtu

Effective

Date

South Dakota

Huron $\quad .0689$

20.18

$12 / 80$

Pierre

9.61

$11 / 80$

Rapid City

.0328

17.08

$11 / 80$

Sioux Falls

.0418

12.25

$11 / 80$

Utah

Bryce Canyon .0583

17.08

$10 / 80$

Cedar City

21.07

.0719

$10 / 80$

Salt Lake City

17.84

$10 / 80$

Vermont

Burlington

.0515

15.09

$11 / 80$

Washington

olympia

.0213

6.24

$11 / 80$

.0139

4.07

$11 / 80$

.0196

5.74

$11 / 80$

.0211

6.18

Richland

.0689

20.18

$11 / 80$

.0192

$11 / 80$

5.63

$11 / 80$

.0157

Tacoma

.0295

4.60

$11 / 80$

8.64

.0213

Whidbey Is land

6.24

Yukinia

.0287

Wi sconsin

Eau CTaire

.0413

.0437

.0413

.0473

La Crosse

Madison

.0455

$11 / 80$

$11 / 80$

8.41

$11 / 80$

Mi lwaukee

12.10

12.80

12.10

13.86

13.33

$11 / 80$

$11 / 80$

$11 / 80$

$11 / 80$

$11 / 80$

Wyoming

Casper

.0287

8.41

$11 / 80$

.0286

8.40

$11 / 80$

Lander

8.41

$11 / 80$

.0287

8.41

$11 / 80$

.0287

8.41

Rock Springs

.0378

$11 / 80$

17.07

$11 / 80$ 